I N S T I T U T O

$\mathrm{DE}$

M E D I C I N A

T R O P I C A L

DE

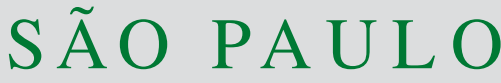

JOURNAL OF THE SÃO PAULO INSTITUTE OF TROPICAL MEDICINE

${ }^{1}$ Universidade de São Paulo, Faculdade de Medicina, Departamento de Moléstias Infecciosas e Parasitárias, São Paulo, São Paulo, Brazil

2Universidade de Sao Paulo, Faculdade de Medicina, Laboratorio de Investigação Médica em Virologia (LIM 52), São Paulo, São Paulo, Brazil

${ }^{3}$ Universidade de São Paulo, Faculdade de Medicina, Instituto de Medicina Tropical de São Paulo, São Paulo, São Paulo, Brazil

${ }^{4}$ Weill Cornel Medicine, Department of Obstetrics and Gynecology, New York, New York, USA

*These authors contributed equally to this study

Correspondence to: Maria Cassia

Mendes-Correa

Universidade de São Paulo, Faculdade de Medicina, Instituto de Medicina Tropical de São Paulo, Av. Dr. Enéas Carvalho de Aguiar, 470, CEP 05403-000, São Paulo, SP, Brazil

E-mail: cassiamc@uol.com.br

Received: 2 February 2022

Accepted: 3 February 2022

\section{Generation of neutralizing antibodies against Omicron, Gamma and Delta SARS-CoV-2 variants following CoronaVac vaccination}

\author{
Almir Ribeiro da Silva Jr. ${ }^{1,2 *}$, Lucy Santos Villas-Boas ${ }^{2 \star}$, Tania Regina \\ Tozetto-Mendoza ${ }^{\circledR 2,3}$, Layla Honorato ${ }^{\circledR}$, Anderson de Paula ${ }^{2}$, Steven S. \\ Witkin $^{1,2,4}$, Maria Cassia Mendes-Correa ${ }^{1,2,3}$
}

\section{ABSTRACT}

Vaccination is a fundamental tool to prevent SARS-CoV-2 infection and to limit the COVID-19 pandemic. The emergence of SARS-CoV-2 variants with multiple mutations has raised serious concerns about the ability of neutralizing antibody responses elicited by prior vaccination to effectively combat these variants. The neutralizing capacity against the Gamma, Delta and Omicron variants of sera from individuals immunized with the CoronaVac vaccine remains incompletely determined. The present study evaluated 41 health care workers at the Faculdade de Medicina of the Universidade de Sao Paulo, in Sao Paulo, Brazil, naive to previous SARS- CoV-2 infection, who were vaccinated with two doses of the CoronaVac SARS-CoV-2 vaccine 28 days apart. Neutralizing antibody levels against the Gamma, Delta, and Omicron variants were measured at 32 and 186 days after the second vaccination. We also measured neutralizing antibodies against Omicron in 34 of these individuals following a subsequent booster immunization with the Pfizer vaccine. Quantification of neutralizing antibodies was performed using the Cytopathic Effect-based Virus Neutralization test. Neutralization antibody activity against the Gamma, Delta and Omicron variants was observed in $78.0 \%, 65.9 \%$ and $58.5 \%$ of serum samples, respectively, obtained at a mean of 32 days after the second immunization. This decreased to $17.1 \%, 24.4 \%$ and $2.4 \%$ of sera having activity against Delta, Gamma and Omicron, respectively, at 186 days post-vaccination. The median neutralizing antibody titers at 32 days were 1:40, 1:20 and 1:20 against Gamma, Delta and Omicron, respectively, and decreased to an undetectable median level against all variants at the later time. A booster immunization with the Pfizer vaccine elicited neutralizing antibodies against Omicron in $85 \%$ of subjects tested 60 days after vaccination. We conclude that two doses of the CoronaVac vaccine results in limited protection of short duration against the Gamma, Delta and Omicron SARS-CoV-2 variants. A booster dose with the Pfizer vaccine induced antibody neutralizing activity against Omicron in most patients which was measurable 60 days after the booster.

KEYWORDS: CoronaVac vaccine. Omicron variant. Gamma variant. Delta variant. Booster.

\section{INTRODUCTION}

SARS-CoV-2 vaccines are an important tool to limit the propagation of COVID-19 and reduce morbidity and mortality. However, the efficacy of these vaccines depends on several factors, including their ability to recognize and neutralize emerging SARS-CoV-2 variants. Evaluating the range of immune responses induced by various SARS-CoV-2 vaccine preparations is crucial for the 
optimization of preventive measures against the disease.

Neutralizing antibodies elicited by previous infection or by vaccination probably represent a key component of protective immunity against SARS-CoV- $2^{1}$. Recently, it has been shown that approximately 20 changes introduced into a synthetic polymutant spike protein was sufficient for substantial evasion of the polyclonal neutralizing antibody response elicited in most persons who had recovered from a COVID-19 infection or had received two doses of a SARSCoV-2 mRNA vaccine ${ }^{1}$.

A new variant of SARS-CoV-2, Omicron (B.1.1.529), was reported by the World Health Organization (WHO) on November 24, 2021 and designated as a variant of concern $^{2}$. It has 32 changes in the amino acid sequence in the spike protein relative to that of the original virus (Wuhan-hu-1), particularly in the receptor-binding domain and the $\mathrm{N}$-terminal domain, the primary targets of neutralizing antibodies ${ }^{3}$. These mutations have the potential for increased transmissibility of the variant coupled with resistance to neutralizing immunity in individuals with a prior SARS-CoV-2 infection or vaccination ${ }^{2}$.

There is still lack of information regarding the neutralization capacity of patients' sera against Omicron and other variants after vaccination with CoronaVac. Also, there is little data on the neutralization capacity against Omicron after a booster dose with the Pfizer vaccine ${ }^{4}$. The objective of our study was to measure neutralizing antibody titers against the Omicron, Delta and Gamma variants in 41 health care personnel, naive to previous SARS-CoV-2 infection, who were vaccinated with the SARS-CoV-2 CoronaVac vaccine (Sinovac Life Sciences, China). We also measured neutralizing antibodies against Omicron in most of these individuals after a booster immunization with the Pfizer mRNA vaccine.

\section{MATERIALS AND METHODS}

\section{Study population}

This prospective study recruited 41 laboratory workers and auxiliary staff from the Instituto de Medicina Tropical de Sao Paulo, at the Faculdade de Medicina of the Universidade de Sao Paulo, Brazil, naive to previous SARS-CoV-2 infection, who were vaccinated with the CoronaVac (Sinovac Life Sciences, Beijing, China) SARS-CoV-2 vaccine according to the Brazilian immunization program established by the Ministry of Health. Vaccination was by intramuscular injection of $3 \mu \mathrm{g}$ in $0.5 \mathrm{~mL}$ of $\beta$-propiolactone-inactivated SARS-CoV-2. Immunization was in two doses 28 days apart administered on January 20 and on February 21, 2021. Exclusion criteria included pregnant women, a previous history of COVID-19, testing positive for antibodies to SARS-CoV-2 on sera collected prior to immunization or prior immunization with any SARS-CoV-2 vaccine. Thirty-four of these individuals (82.9\%) received a booster immunization with the Pfizer mRNA vaccine from September 27 to November 9,2021 . Seven patients were excluded from post-booster sample analysis ( 2 were boosted with other vaccines and blood was not collected from 5 individuals).

\section{Data collection and variables analyzed}

Data was collected using a standardized questionnaire and included age, gender, comorbidities, date of vaccination and quantification of antibodies against the Gamma, Delta and Omicron variants. Serum samples were collected prior to immunization, 30 and 180 days after the second vaccination and 60 days following a booster with the Pfizer vaccine.

\section{Virus isolation}

The Gamma SARS-CoV-2 variant (EPI_ISL_1060902) was obtained as a courtesy from Prof. Ester Sabino. Delta variant (EPI_ISL_2938096) and Omicron variant (EPI_ ISL_6901961) were obtained as a courtesy from Instituto Butantan and from Instituto de Ciencias Biologicas of the Universidade de Sao Paulo, respectively. To isolate SARS-CoV-2 we used Vero cells (ATCC ${ }^{\circledR}$ CCL-81 ${ }^{\mathrm{TM}}$ ). Cells were seeded in a cell culture flask (polystyrene sterile, non-pyrogenic flask, $12.5 \mathrm{~cm}^{2}, 25 \mathrm{~mL}$, Jet Biofil ${ }^{\circledR}$, Guangzhou Jet Bio-Filtration Co., China) in a concentration of $2 \times 10^{5}$ cells $/ \mathrm{mL}$ in $3.0 \mathrm{~mL}$ Dulbecco Minimal Essential Medium (DMEM) supplemented with 5\% heat-inactivated fetal bovine serum (FBS) (Vitrocell Embriolife, Campinas, Brazil) and incubated overnight at $37^{\circ} \mathrm{C}$. The next day, the supernatant was discarded, and $0.5 \mathrm{~mL}$ of a homogenized specimen was added into the culture flask. After $1 \mathrm{~h}$ of incubation (adsorption), we supplemented the volume with $3.0 \mathrm{~mL}$ DMEM containing 2.5\% FBS and $1 \%$ penicillinstreptomycin and inoculated the cultures in a humidified $37^{\circ} \mathrm{C}$ incubator, in an atmosphere of $5 \% \mathrm{CO}_{2}$. We observed for the presence of cytopathic effects (CPE) daily for 3-5 days. The supernatant was collectedand virus replication was confirmed through CPE and by RT-PCR.

The whole genome sequence of the culture isolates was determined using nanopore sequencing (Oxford Nanopore Technologies, Oxonia, Oxford, UK). Purified libraries were sequenced with the Oxford Nanopore MinION device using R9.4.1 flow cells. 


\section{Virus titration}

Twenty-four hours before viral addition, we prepared 96 well sterile polystyrene microtiter plates (Jet Biofil ${ }^{\circledR}$ ) containing $5 \times 10^{4}$ cells $/ \mathrm{mL}$ Vero CCL-81 cells in DMEM. A virus preparation was 10 -fold serially diluted in medium $\left(10^{-1}\right.$ to $\left.10^{-12}\right)$, the original culture medium was then removed and replaced with serial dilutions of the virus in sextuplicate wells and incubated at $37^{\circ} \mathrm{C}$. Observations were performed daily using an inverted light microscope (Nikon Corp 45178, Tokyo, Japan) to verify the presence of CPE over a $72 \mathrm{~h}$ period. The monolayers were then fixed and stained with Naphthol Blue Black (Sigma-Aldrich Co., Saint Louis, Mississippi, USA) dissolved in sodium acetate-acid acetic. The viral titer was expressed in $\mathrm{TCID}_{50} / \mathrm{mL}$ and calculated using the Spearman \& Kärber algorithm, as described by Hierholzer and Killington ${ }^{5}$.

\section{Virus Neutralization Test (VNT)}

The cytopathic effect (CPE)-based virus neutralization test (VNT) was adapted from Nurtop et al. ${ }^{6}$ and was previously described by Wendel et al..$^{7}$ and Mendrone-Junior et al. ${ }^{8}$. VNT was performed with SARS-CoV-2 (EPI ISL_1060902,EPI_ISL_2938096 and EPI_ISL_6901961) in 96-well microtiter plates containing $5 \times 10^{4}$ Vero cells $/ \mathrm{mL}$. Vero cells were seeded in a 96-well microtiter plate and allowed to grow for $24 \mathrm{~h}$ prior to infection. Sera to be tested were heat-inactivated for $30 \mathrm{~min}$ at $56^{\circ} \mathrm{C}$. Then, $110 \mu \mathrm{L}$ of twofold serially diluted sera from 1:20 to 1: 2,560, were added mixed vol/vol with $10^{3} \mathrm{TCID}_{50} / \mathrm{mL}$ of SARS-CoV-2 and incubated at $37{ }^{\circ} \mathrm{C}$ for $1 \mathrm{~h}$ for virus neutralization. The sera-virus mixture was transferred onto the confluent Vero cell monolayer and incubated for $72 \mathrm{~h}$. Cultures at $37^{\circ} \mathrm{C}$ and $5 \% \mathrm{CO} 2$ were observed daily for a CPE. After $72 \mathrm{~h}$, the plates were analyzed by light microscopy (Nikkon, Tokyo, Japan), distinguishing the presence/absence of CPE-VNT. To verify the initial observations after $72 \mathrm{~h}$ the monolayers were fixed and stained with Naphthol Blue Black (SigmaAldrich Co., Saint Louis, Mississippi, USA) dissolved in sodium acetate-acid acetic for $30 \mathrm{~min}$. Dilutions of serum associated with $\mathrm{CPE}$ were considered as a negative result. The absence of CPE or a complete neutralization of SARS-CoV2 inoculum was considered as a positive result. Consequently, the VNT was the highest dilution of serum that neutralized viral growth (absence of a CPE).

For each reaction we used as a positive control diluted viruses in DMEM with $2.5 \% \mathrm{FBS}$ and as a negative control only DMEM with $2.5 \%$ FBS. In addition, a positive control was a serum specimen taken from a patient with a SARS-CoV-2 infection in Sao Paulo, and a negative control was a sample from a patient without neutralizing antibodies, with known VNT results. The antibody titer was calculated as the highest dilution at which CPE was completely inhibited. Titers $\geq 1: 20$ were reported as positive. Virus isolation and VNT were performed in a Biosafety Level 3 laboratory.

\section{Ethics}

This study was approved by the Brazilian National Ethics Review Board (Comissao Nacional de Etica em Pesquisa - CONEP), registry $\mathrm{N}^{\circ}$ 30419620.1.0000.0068. All subjects provided informed written consent.

\section{Statistical analysis}

Qualitative data were described as absolute and relative frequencies and quantitative data were described as summary measures for all individuals. Titers were compared between evaluations for each variant using the paired Wilcoxon test and positivity was compared using the McNemar test. To compare titers for each evaluation between variants we used GEE with a Poisson distribution and logarithm link function, assuming an exchangeable correlation matrix between variants. The analyses were subjected to Bonferroni's multiple comparisons as necessary ${ }^{9,10}$. The titers were compared by GEE with a binomial distribution and log at link function, assuming an exchangeable correlation matrix between variants ${ }^{11}$.

Comparisons of titers between age groups were analyzed by the Mann-Whitney test and the number of positive individuals in each group was evaluated by the Fisher exact test ${ }^{11-13}$. The analyzes were performed using the software IBM-SPSS for Windows version 22.0 (IBM Corp., Armonk, New York, USA) and tabulated using the Microsoft-Excel 2010 software. All tests were performed with a $5 \%$ significance level.

\section{RESULTS}

Between December 2020 and January 2021, 41 eligible individuals agreed to participate in this investigation and had blood samples collected. The time interval between analysis for IgG antibody against SARS-CoV-2 and the initial CoronaVac vaccination was 5 days. The median time (range) from receiving the second dose of CoronaVac to the first or second blood collection for VNT was 32 days (21-81) and 186 days (180-206), respectively. The median time from receiving the second dose of CoronaVac to receiving the Pfizer vaccine booster was 230 (192-246) days and the third blood collection was after another 
61 (21-113) days. Most individuals (90.2\%) were female and the subjects' age ranged from 30 to 77 , with a median of 54 years. The majority $(65.9 \%)$ were $<60$ years old. The mean intervals from the second vaccination to blood collections for analysis were a mean (SD) of 32.1 (12.8) and 186.8 (6.7) days, respectively.

The number of post-vaccination sera with virus neutralization activity and the virus neutralizing titer (VNT) against the SARS-CoV-2 variants at different time points is shown in Table 1. At 32 days after vaccination $78.0 \%$ of the sera neutralized the Gamma variant, $65.9 \%$ neutralized the Delta variant and $58.5 \%$ neutralized the Omicron variant. These differences were not significant. Subsequently, at 186 days following vaccination $17.1 \%$, $24.4 \%$ and $2.4 \%$ of the sera neutralized the Gamma, Delta and Omicron variants, respectively. The difference in the number of sera positive for neutralizing activity against the Gamma and Omicron variants was significant $(\mathrm{p}=0.007)$. The VNT was higher for all three variants at 32 days than at 186 days post-vaccination $(\mathrm{p}<0.001)$ and was the highest at the earlier time point for the Gamma variant.

Sixty days following the Pfizer vaccine booster, neutralizing antibodies against Omicron were present in $85 \%$ of the 34 subjects tested.

The number of positive sera and the VNT against each variant as a function of subjects' age ( $<60 \mathrm{vs.} \geq 60)$ is shown in Table 2. The number of neutralizing sera $(p=0.004)$ as well as the neutralizing titers $(\mathrm{p}=0.003)$ were significantly higher in the younger as compared to the older age group only for the Gamma variant.

Table 1 - Presence and titer of neutralizing activity against SARS-CoV-2 variants in sera from individuals following vaccination with the Coronavax vaccine and booster with the Pfizer vaccine.

\begin{tabular}{lccc}
\hline Variant & $\begin{array}{c}\text { Mean days } \\
\text { Post-vaccination }\end{array}$ & $\begin{array}{c}\text { No of individuals with } \\
\text { neutralizing activity (\%) }\end{array}$ & $\begin{array}{c}\text { Median VNT } \\
\text { (minimum, maximum) }\end{array}$ \\
\hline Gamma & 32.1 & $32(78.0 \%)$ & $1: 40(0,1: 1,280)$ \\
Delta & 32.1 & $27(65.9 \%)$ & $1: 20(0,1: 80)$ \\
Omicron & 32.1 & $24(58.5 \%)$ & $1: 20(0,1: 40)$ \\
Gamma & 186.8 & $10(17.1 \%)$ & $0(0,1: 80)$ \\
Delta & 186.8 & $7(24.4 \%)$ & $0(0,1: 80)$ \\
Omicron & 186.8 & $1(2.4 \%)$ & $0(0,1: 20)$ \\
Omicron & 62.7 & $29(85 \%)$ & $1: 40(0,1: 160)$ \\
\hline
\end{tabular}

Sera from individuals previously immunized against SARS-CoV-2 with the CoronaVax vaccine were analyzed for their virus neutralization titer (VNT) against three different SARS-CoV-2 variants. The presence of virus neutralization activity was defined as the absence of cytopathic activity at a serum dilution $\geq 1: 20$. Differences between the number and titer of positive sera in the first and second blood sample for each variant were significant $(p<0.001)$, as was the difference in the number of sera with VNT against the Gamma and Omicron variants at 186.8 days $(p=0.007)$. Analyses were by the Fisher exact test and the paired Wilcoxon test.

Table 2 - Influence of age on neutralization of SARS-CoV-2 variants at three time points in individuals vaccinated with the CoronaVac vaccine and booster with the Pfizer vaccine.

\begin{tabular}{|c|c|c|c|c|c|}
\hline \multirow{2}{*}{ Analysis } & \multirow{2}{*}{ Timepoint } & \multirow{2}{*}{ Variant } & $<60$ years old & $\geq 60$ years old & \multirow{2}{*}{$p$-value } \\
\hline & & & $N=27$ & $\mathrm{~N}=14$ & \\
\hline & & Gamma & $1: 40(0,1: 1,280)$ & $1: 10(0,1: 80)$ & 0.003 \\
\hline \multirow[t]{3}{*}{ VNT } & one & Delta & $1: 20(0,1: 80)$ & $1: 20(0,1: 40)$ & 0.246 \\
\hline & & Omicron & $1: 20(0,1: 40)$ & $1: 10(0,1: 40)$ & 0.269 \\
\hline & & Gamma & $0(0,1: 80)$ & $0(0,1: 20)$ & 0.391 \\
\hline \multirow[t]{3}{*}{ VNT } & two & Delta & $0(0,1: 80)$ & $0(0,0)$ & 0.185 \\
\hline & & Omicron & $0(0,1: 20)$ & $0(0,0)$ & 0.860 \\
\hline & & Gamma & 25 (92.6\%) & $7(50.0 \%)$ & 0.004 \\
\hline \multirow[t]{3}{*}{ No. positive (\%) } & one & Delta & 19 (70.4\%) & $8(57.0 \%)$ & 0.494 \\
\hline & & Omicron & $17(63.0 \%)$ & $7(50.0 \%)$ & 0.512 \\
\hline & & Gamma & $8(29.6 \%)$ & $2(14.3 \%)$ & 0.447 \\
\hline \multirow[t]{2}{*}{ No. positive (\%) } & two & Delta & $7(25.9 \%)$ & $0(0)$ & 0.075 \\
\hline & & Omicron & $1(3.7 \%)$ & $0(0)$ & $>0.999$ \\
\hline $\mathrm{N}^{\text {o. }}$ positive (\%) & three & Omicron & $20(87 \%)$ & $9(81.8 \%)$ & $>0.999$ \\
\hline
\end{tabular}

Timepoint one has a mean (SD) of 32.1 (12.8) days after vaccination. Timepoint two has a mean (SD) of 186.8 (6.7) days after vaccination. Timepoint three has a mean (SD) 62.7 (20.7) days after the booster (Pfizer). Differences in VNT were determined by the Mann-Whitney test. Differences in the $\mathrm{n}^{\circ}$. of positive subjects were analyzed by the Fisher exact test. 


\section{DISCUSSION}

Vaccine-induced protective immunity has been mainly attributed to the generation of neutralizing antibodies that block virus entry into host target cells ${ }^{12,13}$. A major concern has been limitations in the magnitude, protective capacity and duration of these antibody responses against viral variants after administration of different SARS-CoV-2 vaccines $^{12-14}$. CoronaVac, as well as all current Covid-19 vaccines, are based on a wild-type SARS-CoV-2 isolate that was sequenced in January $2020^{1}$. The present communication reinforces these concerns. The in vitro virus neutralization activity against the Gamma, Delta and Omicron SARS-CoV-2 variants in sera from CoronaVac recipients was of low titer and short duration. However, a subsequent immunization with the Pfizer vaccine enhanced the neutralization titer against Omicron 60 days after booster administration.

A few recent studies investigated the duration and maintenance of neutralizing antibody responses against the Omicron, Delta and Gamma variants after the administration of different SARS-CoV-2 vaccines ${ }^{16,17}$ Overall, the Omicron variant exhibited an unprecedented degree of immune evasion after one or two doses of different vaccines ${ }^{3,15-20}$. Our findings parallel these reports. The number of Coronavac-vaccinated individuals and the virus neutralization titer was lower against the Omicron variant than against the Gamma and Delta variants. The limited number of studies that have evaluated neutralizing antibody titers in sera from participants who received CoronaVac vaccine consistently observed that the titers against Omicron significantly decreased over time $e^{4,16,21}$ However, the rate of decrease in antibody levels and/or the detection of neutralizing antibody differed among the studies after CoronaVac vaccination. In our study, $58 \%$ of vaccinated individuals had neutralizing antibody activity against Omicron 30 days after administration of the second dose. These differences may be due to the time interval between sample collection and SARS-CoV-2 vaccination. To the best of our knowledge, we are the only group to study neutralizing antibody activity 30 and 180 days after vaccination with CoronaVac. Other published studies evaluated neutralizing activity at shorter time intervals.

In our study, vaccine-induced immune protection against the Gamma and Delta variants was also of short duration after two doses of the CoronaVac vaccine. These observations parallel findings from other studies that analyzed neutralizing activity against these variants after CoronaVac or other vaccines ${ }^{16}$.

Advantages of our study include utilization of a live virus neutralization assay, acknowledged as the gold standard ${ }^{22}$, to quantitate anti-viral activity. Also, our study population was verified to be naïve to previous SARS-CoV- 2 infection, which reinforces the validity of the data obtained. We also acknowledge limitations. Although our findings are in agreement with previous reports, the limited sample size of our study requires an expanded study on a larger number of individuals to verify our observations. We also assessed neutralizing antibody titers only at two-time intervals after the second vaccination, and so further studies are needed to better define the rate of decay of protective immunity over time. Our study measured viral neutralization in an in vitro model. We did not measure cell-mediated immune components of the anti-viral immune response that also contributes to viral neutralization in vivo. Thus, our findings may not completely parallel the actual anti-viral activity present against each variant in our subjects.

The CoronaVac vaccine has been available to the adult Brazilian population since January 2021 and was the first option provided to health care workers at our institution. Omicron was first described in December 2021. It is plausible that the recent increase in COVID-19 in Brazil might, in large part, be associated with the poor neutralizing activity of sera from CoronaVac recipients who received the two-dose vaccine schedule. Our data reinforces that there is an urgent need to evaluate current vaccine strategies for CoronaVac recipients specifically, as well as for recipients of other vaccines. Development of newer generation vaccines in addition to effective booster dose strategies are necessary to maximize prevention and minimize transmission of SARS-CoV-2 infection and its consequences.

\section{CONCLUSION}

Individuals vaccinated with CoronaVac exhibit a significant loss of neutralizing antibodies against the Omicron, Delta and Gamma variants 186 days after receiving their second immunization. A booster dose with the Pfizer vaccine induced antibody neutralizing activity against Omicron in most patients which was measurable 60 days after the booster.

\section{ACKNOWLEDGMENTS}

We thank all participants from Instituto de Medicina Tropical de Sao Paulo from whom the pre and postimmunization sera were obtained. We thank the many colleagues and staff from LIM-52 (Virology Laboratory), at Instituto de Medicina Tropical de Sao Paulo who participated in all phases of the study. 


\section{AUTHORS' CONTRIBUTIONS}

Conceptualization, writing and review, and supervision: ARSJ, LSVB, MCMC and SSW; data curation, laboratory assays, analysis and interpretation of data: ARSJ, TRTM, LH, AP and LSVB; formal analysis: MCMC and SSW; funding acquisition: MCMC. All authors approved the final version of the manuscript.

\section{CONFLICT OF INTERESTS}

The authors declare that they have no conflict of interests.

\section{FUNDING}

MCMC receives a scholarship from $C N P q$ - Conselho Nacional de Desenvolvimento Cientifico e Tecnológico for the purpose of conducting scientific research: process $\mathrm{N}^{\circ}$ 309492/2018-1.

\section{REFERENCES}

1. Schmidt F, Weisblum Y, Rutkowska M, Poston D, Da Silva J,Zhang F, et al. High genetic barrier to SARS-CoV-2 polyclonal neutralizing antibody escape. Nature 2021;600:512-6.

2. CDC COVID-19 Response Team. SARS-CoV-2 B.1.1.529 (Omicron) Variant - United States, December 1-8, 2021. MMWR Morb Mortal Wkly Rep. 2021;70:1731-4.

3. Schmidt F, Muecksch F, Weisblum Y, Da Silva J, Bednarski E, Cho A, et al. Plasma neutralization of the SARS-CoV-2 Omicron variant. N Engl J Med. 2021 In Press.

4. Pérez-Then E, Lucas C, Monteiro VS, Miric M, Brache V, Cochon L, et al. Neutralizing antibodies against the SARS-CoV-2 Delta and Omicron variants following heterologous CoronaVac plus BNT162b2 booster vaccination. Nat Med. 2022 In Press.

5. Hierholzer JC, Killington RA. Virus isolation and quantitation. In: Mahy B, Kangro H, editors. Virology methods manual. San Diego: Academic Press; 1996. p.25-46.

6. Nurtop E, Villarroel PM, Pastorino B, Ninove L, Drexler JF, Roca Y et al. Combination of ELISA screening and seroneutralisation tests to expedite Zika virus seroprevalence studies. Virol J. 2018;15:192.

7. Wendel S, Kutner JM, Machado R, Fontão-Wendel R, Bub C, Fachini R, et al. Screening for SARSCoV-2 antibodies in convalescent plasma $(\mathrm{CCP})$ in Brazil: preliminary lessons from a voluntary convalescent donor program. Transfusion. 2020;60:2938-51.

8. Mendrone-Junior A, Dinardo CL, Ferreira SC, Nishya A, Salles NA, Almeida Neto C, et al. Correlation between SARS-COV-2 antibody screening by immunoassay and neutralizing antibody testing. Transfusion. 2021;61:1181-90.

9. Kirkwood BR, Sterne JA. Essential medical statistics. $2^{\text {nd }}$ ed. Massachusetts: Blackwell Science; 2003.

10. Mccullagh AG, Nelder JA. Generalized linear models. $2^{\text {nd }}$ ed. New York: Chapman and Hall; 1989.

11. Neter J, Kutner MH, Nachtsheim CJ, Wasserman W. Applied linear statistical models. $4^{\text {th }}$ ed. Ilinois: Richard D. Irwing; 1996.

12. Dong Y, Dai T, Wei Y, Zhang L, Zheng M, Zhou F. A systematic review of SARS-CoV-2 vaccine candidates. Signal Transduct Target Ther. 2020;5:237.

13. Naaber P, Tserel L, Kangro K, Sepp E, Jürjenson V, Adamson A, et al. Dynamics of antibody response to BNT162b2 vaccine after six months: a longitudinal prospective study. Lancet Reg Health Eur. 2021;10:100208.

14. Cevik M, Grubaugh ND, Iwasaki A, Openshaw P. COVID-19 vaccines: keeping pace with SARS-CoV-2 variants. Cell. 2021;184:5077-81.

15. Dejnirattisai W, Shaw RH, Supasa P, Liu C, Stuart AS, Pollard $\mathrm{AJ}$, et al. Reduced neutralisation of SARS-CoV-2 omicron B.1.1.529 variant by post-immunisation serum. Lancet. 2022;399:234-6.

16. Wang Y, Ma Y, Xu Y, Liu J, Li X, Chen Y, et al. Resistance of SARS-CoV-2 Omicron variant to convalescent and CoronaVac vaccine plasma. Emerg Microbes Infect. 2022;11:424-7.

17. Ai J, Zhang H, Zhang Y, Lin K, Zhang Y, Wu J, et al. Omicron variant showed lower neutralizing sensitivity than other SARS$\mathrm{CoV}-2$ variants to immune sera elicited by vaccines after boost. Emerg Microbes Infect. 2022;11:337-43.

18. Lusvarghi S, Pollett SD, Neerukonda SN, Wang W, Wang R, Vassell R, et al. SARS-CoV-2 Omicron neutralization by therapeutic antibodies, convalescent sera, and post-mRNA vaccine booster. bioRxiv. 2021 In Press.

19. Syed AM, Ciling A, Khalid MM, Sreekumar B, Chen PY, Kumar GR, et al. Omicron mutations enhance infectivity and reduce antibody neutralization of SARS-CoV-2 virus-like particles. medRxiv. 2022 In Press.

20. Cheng SM, Mok CK, Leung YW, Ng SS, Chan KC, Ko FW, et al. Neutralizing antibodies against the SARS-CoV-2 Omicron variant following homologous and heterologous CoronaVac or BNT162b2 vaccination. Nat Med. 2022 In Press.

21. Lu L, Mok BW, Chen LL, Chan JM, Tsang OT, Lam BH, et al. Neutralization of SARS-CoV-2 Omicron variant by sera from BNT162b2 or Coronavac vaccine recipients. Clin Infect Dis. 2021 In Press.

22. Pang NY, Pang AS, Chow VT, Wang DY. Understanding neutralizing antibodies against SARS-CoV-2 and their implications in clinical practice. Mil Med Res. 2021;8:47. 